\title{
RESIDUATED STRUCTURES DERIVED FROM COMMUTATIVE IDEMPOTENT SEMIRINGS
}

\author{
IvAN ChAJdA $^{a}$ AND Helmut LÄNGER ${ }^{a, b}$ \\ ${ }^{a}$ Palacký University Olomouc \\ Faculty of Science, Department of Algebra and Geometry \\ 17. listopadu 12, 77146 Olomouc, Czech Republic \\ ${ }^{b}$ TU Wien, Faculty of Mathematics and Geoinformation \\ Institute of Discrete Mathematics and Geometry \\ Wiedner Hauptstraße 8-10, 1040 Vienna, Austria \\ e-mail: ivan.chajda@upol.cz \\ helmut.laenger@tuwien.ac.at
}

\begin{abstract}
Since the reduct of every residuated lattice is a semiring, we can ask under what condition a semiring can be converted into a residuated lattice. It turns out that this is possible if the semiring in question is commutative, idempotent, G-simple and equipped with an antitone involution. Then the resulting residuated lattice even satisfies the double negation law. Moreover, if the mentioned semiring is finite then it can be converted into a residuated lattice or join-semilattice also without asking an antitone involution on it. To a residuated lattice $\mathbf{L}$ which does not satisfy the double negation law there can be assigned a so-called augmented semiring. This can be used for reconstruction of the so-called core $C(\mathbf{L})$ of $\mathbf{L}$. Conditions under which $C(\mathbf{L})$ constitutes a subuniverse of $\mathbf{L}$ are provided.

Keywords: semiring, commutative, idempotent, G-simple, antitone involution, commutative residuated lattice, commutative residuated join-semilattice, divisible, prelinear, double negation law.
\end{abstract}

2010 Mathematics Subject Classification: 06B99, 16Y60, 03 G25.

Support of the research by ÖAD, project CZ 04/2017, support of the research of the first author by IGA, project PřF 2018 012, and support of the research of the second author by the Austrian Science Fund (FWF), project I 1923-N25 entitled "New perspectives on residuated posets", is gratefully acknowledged. 


\section{REFERENCES}

[1] L.P. Belluce, A. Di Nola and A.R. Ferraioli, $M V$-semirings and their sheaf representations, Order 30 (2013) 165-179. doi:10.1007/s11083-011-9234-0

[2] R. Bělohlávek, Fuzzy Relational Systems, Foundations and Principles (Kluwer, New York, 2002). ISBN 0-306-46777-1/hbk.

[3] G. Birkhoff, Lattice Theory, AMS (Providence, R.I., 1979). ISBN 0-8218-1025-1.

[4] I. Chajda, A representation of residuated lattices satisfying the double negation law, Soft Computing 22 (2018) 1773-1776. doi:10.1007/s00500-017-2673-9

[5] I. Chajda and H. Länger, When does a semiring become a residuated lattice?, AsianEur. J. Math. 9 (2016) 1650088 (10 pages). doi:10.1142/S1793557116500881

[6] R.P. Dilworth, Non-commutative residuated lattices, Trans. Amer. Math. Soc. 46 (1939) 426-444. doi:10.2307/1989931 https://www.jstor.org/stable/1989931.

[7] N. Galatos, P. Jipsen, T. Kowalski and H. Ono, Residuated Lattices: An Algebraic Glimpse at Substructural Logics (Elsevier, Amsterdam, 2007). ISBN 978-0-444-52141-5.

[8] J.S. Golan, The Theory of Semirings with Applications in Mathematics and Theoretical Computer Science (Longman, Harlow, 1992). ISBN 0-582-07855-5.

[9] P. Jipsen, From semirings to residuated Kleene lattices, Studia Logica 76 (2004) 291-303. doi:10.1023/B:STUD.0000032089.54776.63

[10] J. Pavelka, On fuzzy logic, II Enriched residuated lattices and semantics of propositional calculi, Z. Math. Logik Grundlag. Math. 25 (1979) 119-134. doi:10.1002/malq.19790250706

[11] C. Tsinakis and A.M. Wille, Minimal varieties of involutive residuated lattices, Studia Logica 83 (2006) 407-423. doi:10.1007/s11225-006-8311-7

[12] M. Ward and R.P. Dilworth, Residuated lattices, Trans. Amer. Math. Soc. 45 (1939) $335-354$. doi:10.1090/S0002-9947-1939-1501995-3

[13] A.M. Wille, A Gentzen system for involutive residuated lattices, Algebra Universalis 54 (2005) 449-463. doi:10.1007/s00012-005-1957-6

Received 24 June 2017

Revised 17 June 2018 Accepted 9 December 2018 